\title{
Fixing Balanced Knockout and Double Elimination Tournaments
}

\author{
Haris Aziz, Serge Gaspers \\ Data61, CSIRO and UNSW Sydney, Australia \\ Simon Mackenzie \\ Carnegie Mellon University, USA \\ Nicholas Mattei \\ IBM Research, USA \\ Paul Stursberg \\ Technische Universität München, Germany \\ Toby Walsh \\ Data61, CSIRO and UNSW Sydney, Australia
}

\begin{abstract}
Balanced knockout tournaments are one of the most common formats for sports competitions, and are also used in elections and decision-making. We consider the computational problem of finding the optimal draw for a particular player in such a tournament. The problem has generated considerable research within $\mathrm{AI}$ in recent years. We prove that checking whether there exists a draw in which a player wins is NP-complete, thereby settling an outstanding open problem. Our main result has a number of interesting implications on related counting and approximation problems. We present a memoization-based algorithm for the problem that is faster than previous approaches. Moreover, we highlight two natural cases that can be solved in polynomial time. All of our results also hold for the more general problem of counting the number of draws in which a given player is the winner. Finally, we show that our main NP-completeness result extends to a variant of balanced knockout tournaments called doubleelimination tournaments.
\end{abstract}

\footnotetext{
This is a revised and expanded version of Aziz et al. [3] with additional proof details as well as new results for tournaments with kings and double elimination tournaments.

Email addresses: haris.aziz@data61.csiro.au (Haris Aziz), serge.gaspers@data61.csiro.au (Serge Gaspers), simonm@andrew.cmu.edu (Simon Mackenzie), N.Mattei@ibm.com (Nicholas Mattei), paul.stursberg@ma.tum.de (Paul Stursberg), toby.walsh@data61.csiro.au (Toby Walsh)
} 
Keywords: Knockout tournaments, Seedings of a tournament, Computational Complexity

JEL: $\mathrm{C} 62$, C63, and $\mathrm{C} 78$

\section{Introduction}

Balanced knockout tournaments are one of the most widely-used formats for sports competitions $[7,10,13]$. A prominent example is the Wimbledon Men's Singles tennis tournament in which 128 players enter the tournament and the player who wins seven consecutive matches right from the first round to the final wins the tournament. The format is also used in certain elimination style election and decision making schemes and has received considerable interest in the field of artificial intelligence $[12,16,17,22,32,34,35,25,1]$ as well as social sciences [5, 20, 21, 23, 31]. Knockout tournaments which are balanced are of particular interest, as they are considered to be fair [19] and allow a large number of matches to be played in parallel.

Consider the setting in which there is a set of players $N=[n]$ (we use the notation $[n]:=\{1, \ldots, n\})$ where $n=2^{c}$ for some integer $c{ }^{1}$ Given $N$, an ordered balanced knockout tournament $\mathrm{T}(N, \pi)$ is defined as a balanced binary tree with $n$ leaf nodes where the seeding $\pi$ specifies the labelling of the leaf nodes with respect to $N$. All ordered balanced knockout tournaments that are isomorphic to each other (with respect to the labelling of the leaf nodes) are said to have the same draw. They are represented by a single (unordered) balanced knockout tournament (BKT) $\mathrm{T}(N, \sigma)$ where $\sigma$ denotes the draw. The set of all draws is denoted by $\Sigma$. Whereas the total number of seedings is $n$ !, the number of draws is $\frac{n !}{2^{n-1}}$ as all pairwise matchups in the leaf nodes are the same if adjacent elements of the seeding are swapped, but even this grows very rapidly. For a tournament like Wimbledon, $n=128$ and the number of distinct draws is $\approx 2.2665 \cdot 10^{177}$. This is significantly more than the number of atoms in the universe, or even a googol.

A BKT $\mathrm{T}(N, \sigma)$ is conducted in the following fashion. Players that correspond to sibling leaf nodes play a match against each other. The winner of the match proceeds up the tree to the next round. The winner of $\mathrm{T}(N, \sigma)$ is the player who reaches the root node. We are given a pairwise comparison matrix $P$ such that $P_{i j} \in[0,1]$ denotes the probability of player $i$ beating player $j$ in a pairwise elimination match and $0 \leq P_{i j}=1-P_{j i} \leq 1$. We call $P$ deterministic if $P_{i j} \in\{0,1\}$ for all players $i, j \in N$. In this case, we say that player $i$ beats player $j$ if $P_{i j}=1$. Given $N, P$ and a draw $\sigma$, each player $i \in N$ has a certain probability $\operatorname{wp}(i, N, P, \sigma)$ of being the winner of $\mathrm{T}(N, \sigma)$. This probability can be computed in time $O\left(n^{2}\right)$ via a recursive formulation [34]. We denote by

${ }^{1}$ The setting is general enough to cover the case where some players get byes in the first round. In that case we can consider a dummy player who always loses to the player who gets a bye. 
$\operatorname{mwp}(i, N, P):=\max _{\sigma \in \Sigma}(\operatorname{wp}(i, N, P, \sigma))$ the maximum possible winning probability of $i$ in $\mathrm{T}(N, \sigma)$ taken over all draws $\sigma \in \Sigma$.

We can now define the Probabilistic Tournament Fixing Problem (PTFP) in which the probability of each player beating another player is known and the goal is to find a draw that maximizes the probability of a certain player winning the BKT.

\section{Probabilistic Tournament Fixing Problem (PTFP)}

Instance: Player set $N$, pairwise comparison matrix $P$, a distinguished player $i^{*} \in N$, and target probability $q \in[0,1]$.

Question: Does there exist a draw $\sigma$ for the player set $N$ for which the probability of $i^{*}$ winning $\mathrm{T}(N, \sigma)$ is at least $q$ ?

PTFP was proposed by $\mathrm{Vu}$ et al. [34] and has been studied in numerous papers (see e.g., $[27,28,29]$ ). It is a well-motivated problem in sports analytics [26]. PTFP has been shown to be NP-hard for various restrictions, including the case where the entries of $P$ are restricted to $\{0,1 / 2,1\}$ [34] and the case where the matrix $P$ is deterministic and certain matches are not allowed [32].

Nevertheless, the computational complexity of a particularly natural and interesting special case, the Tournament Fixing Problem (TFP), has remained a major open question. In the TFP, the matrix $P$ is deterministic and all matches are allowed. The winner of each match is deterministically known beforehand and the question is whether there exists a draw for which a given player wins in the corresponding BKT.

\section{Tournament Fixing Problem (TFP)}

Instance: Player set $N$, deterministic pairwise comparison matrix $P$, and a distinguished player $i^{*} \in N$.

Question: Does there exist a draw $\sigma$ for the player set $N$ for which $i^{*}$ is the winner of $\mathrm{T}(N, \sigma)$ ?

TFP is equivalent to checking whether there exists a seeding $\pi$ for which $i^{*}$ is the winner of $\mathrm{T}(N, \pi)$. We note that TFP is a special case of the problem with the same name as defined in Vassilevska Williams [32], where there can be additional constraints by which certain matches are disallowed. TFP is also a special case of \#TFP - the problem of counting the number of draws for which a given player is the winner. This count can be used to compute the probability of a player winning in a draw chosen uniformly at random. It can also be interpreted as the relative strength of the player.

Contributions. We first settle the computational complexity of TFP by showing that it is NP-complete. The question was explicitly stated as an open problem a number of times $[12,18,33,24,27,28,29,32,34,35]$. As a corollary, we show that unless $\mathrm{P}=\mathrm{NP}$, there exists no polynomial-time approximation algorithm for computing the maximal winning probability of a player. This inapproximability result provides additional motivation for the line of work in which heuristic 
algorithms have been proposed for PTFP [35]. Another corollary is that there exists no fully polynomial time randomized approximation scheme (FPRAS) for counting the number of draws for which a player is the winner.

In view of these intractability results, we identify two natural cases for which even \#TFP (and hence also TFP) can be solved in polynomial time. In the first case, the players can be divided into a constant number of player types. This setting appeals to the scenario where players can be divided into groups based on similar intrinsic ability. In the second case, there is a linear ordering on the ability of players with a constant number of exceptions where a player with lower ability beats a player with higher ability. ${ }^{2}$ Finally, we provide an exact memoization-based algorithm to solve \#TFP that is faster than known exact approaches to solve the problem: it runs in time $O\left(2.8285^{n}\right)$ and uses space $O\left(1.7548^{n}\right)$. If only polynomial space is available, the running time becomes $4^{n+o(n)}$, and we give a range of possible time-space trade-offs.

Finally, we consider double-elimination tournaments which are a variant of knockout tournaments in which the losers get a second chance to win the overall tournament. We show that TFP is NP-complete for this problem as well thereby answering another open problem [30].

Related Work. After the work of Vu et al. [34], PTFP and TFP have been studied in a number of research papers. Vassilevska Williams [32] identified various sufficient conditions for a player to be a winner of a BKT, e.g. if he is a king who beats half of all players. In a followup paper, Stanton and Vassilevska Williams [29] focused on when weak players can possibly win a BKT.

The authors of this paper proved Theorem 1 in a conference paper [3] and provided two additional tractable cases as well as an exponential-time algorithm. Subsequently, Kim and Vassilevska Williams [17] showed that TFP remains NP-hard if the distinguished player is a king that beats $n / 4$ players. They also prove that TFP remains NP-hard for 3 -kings who can beat $n / 2$ players. These structural results about kings are enclosed in one general theorem in Kim et al. [16]. Furthermore, Kim and Vassilevska Williams [17] present an algorithm that improves the (exponential) running time of that in Aziz et al. [3], albeit at the expense of requiring exponential space.

Stanton and Vassilevska Williams [27, 28] identified conditions in a probabilistic model under which the tournament organizer can fix the tournament with high probability. In $\mathrm{Vu}$ and Shoham [36], the problem of designing 'fair' draws was considered. Lang et al. [19] and Lang et al. [18] examined winner determination in voting trees that need not be balanced. In more recent work, Chatterjee et al. [6] examine the robustness of winning probability with respect

\footnotetext{
${ }^{2}$ The condition is quite natural since in many competitions there is a clear-cut ranking of the players according to their skills with only a few pairs of players for which the weaker player can beat the stronger player. For example, as of 15/01/2014, Nikolay Davydenko was the only tennis player among the men's top 64 who had a winning head-to-head record against Rafael Nadal. Russell and van Beek [24] provide an extended discussion and empirical data on this phenomena.
} 
to small errors in the pairwise winning probabilities.

TFP can also be considered as the problem of checking whether a player is a possible winner in a deterministic BKT. Computing possible winners for other voting rules where the information on the preferences is not complete has been studied extensively $[2,38]$. Another related problem is checking whether a sports team can still win a round-robin competition when all the matches have not yet been completed $[11,15]$.

\section{TFP is NP-complete}

In this section, we settle the complexity of TFP. For convenience, we will represent the pairwise comparison matrix $P$ as a directed graph where an edge from $i$ to $j$ exists iff $i$ beats $j$.

Theorem 1. TFP is NP-complete.

Proof. We reduce from the NP-hard variant of the 3SAT problem in which every literal appears at most twice [4]. Given such a 3SAT instance $F=(X, C)$ where $X=\left\{x_{1}, \ldots, x_{|X|}\right\}$ is the set of variables and $C$ the set of clauses, we build an instance of TFP with a distinguished player who can win the tournament by some draw if and only if $F$ is satisfiable.

The TFP instance consists of a set of players $N=\{1, \ldots, n\}$ where $n$ is the smallest power of 2 greater than or equal to $32 \cdot|X|$. The resulting knock-out tournament will thus consist of $R:=\log (n)=\lceil\log (32 \cdot|X|)\rceil \geq 5$ rounds where the first (lowest) four rounds will be used to store the gadgets while the later rounds will enforce certain outcomes for the gadgets.

In the following, we use the notation $A \dot{\cup} B$ for the union of two sets $A$ and $B$ which are disjoint, i.e. $A \cap B=\emptyset$. We can decompose the set of players $N$ as follows

$$
N=M \dot{\cup} S \dot{\cup} G^{\mathrm{X}} \dot{\cup} G^{\mathrm{CG}} \dot{\cup} G^{\mathrm{F}}
$$

where players in $G^{\mathrm{X}}$ are used in the choice gadgets that will model the variable assignment, players in $G^{\mathrm{CG}}$ are used in the clause/garbage gadgets that will model the behaviour of the clauses, and players in $G^{\mathrm{F}}$ are used in filler gadgets that will be used to balance the BKT. Players in $S$ are special players that will ensure the connection between choice and clause gadgets.

There will be a total of $k:=\frac{n}{16}$ gadgets, each associated with one of the players from the set $M=\left\{m_{1}, m_{2}, \ldots, m_{k}\right\}$. The player $m_{1}$ will be our distinguished player. We will show that for $m_{1}$ to win the BKT, all players from $M$ will have to proceed to the fifth round. The structure of the gadgets will make sure that this can happen if and only if the 3SAT instance $F$ is satisfiable.

More precisely, we use $|X|$ choice gadgets numbered from 1 to $|X|,|X|$ clause/garbage gadgets (that may each model up to two clauses) numbered from $|X|+1$ to $2|X|$ and $(k-2|X|)$ filler gadgets numbered from $(2|X|+1)$ to $k$. As every literal may appear at most twice, note that the number of clauses is bounded by $|C| \leq \frac{4|X|}{3} \leq 2|X|$ and hence the $|X|$ clause/garbage gadgets provide enough space to model all clauses in $C$. 


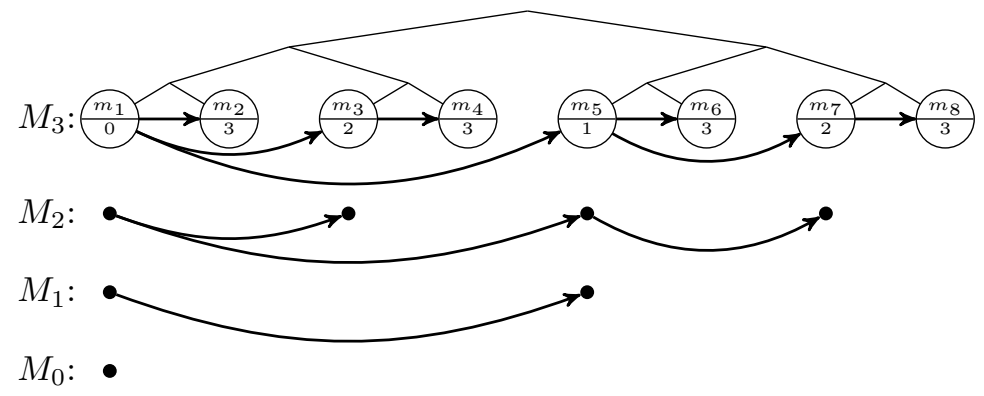

Figure 1: The spawning process for the case $k=8$, the lower part of each node shows the corresponding value of $l_{j}$. By the draw shown on top of $M_{3}$, the leftmost player can win the BKT.

Using this numbering, we can further partition the above sets as follows:

$$
G^{\mathrm{X}}=\bigcup_{j=1}^{|X|} G_{j} ; \quad G^{\mathrm{CG}}=\bigcup_{j=|X|+1}^{2|X|} G_{j} ; \quad G^{\mathrm{F}}=\bigcup_{j=2|X|+1}^{k} G_{j}
$$

Note that the sets $G_{j}$ have 10,13 or 15 elements, depending on whether they are a subset of $G^{\mathrm{X}}, G^{\mathrm{CG}}$ or $G^{\mathrm{F}}$, respectively.

Set $\boldsymbol{M}$, the Spawning Process. The relation between elements of the set of winners $M$ is recursively defined via a linear ordering of players as follows: We start with player $m_{1}$. At each iteration, every player spawns a new player placed directly to his right. In the pairwise comparison graph, each player beats all players to his left in this construction except for the one that spawned him. This recursive construction is repeated until a total of $k$ players are present. We denote by $M_{\ell}$ the set of $2^{\ell}$ players from $M$ that are present after the $\ell$-th iteration of the spawning process. Furthermore, for any $j \in[k]$, let $\ell_{j}$ be the smallest $\ell$ such that $m_{j} \in M_{\ell}$ (see Figure 1).

Lemma 1. There is a draw $\sigma$ such that $m_{1}$ wins the BKT $T(M, \sigma)$.

Proof. Seeding all players in $M$ from left to right according to the spawning process makes $m_{1}$ win the tournament (see Figure 1): Whenever two players meet, the left player in the match has spawned the right player, thus the left player wins. As the leftmost player, $m_{1}$ wins the tournament.

We will show later that this is the only way for $m_{1}$ to win the tournament. In other words, $m_{1}$ can win the tournament if and only if all players from $M$ can reach the fifth round simultaneously.

Global structure. We now describe how the sets from (1) and (2) relate to each other. In general it is true that players from $S$ beat all other players and players from $G^{\mathrm{X}} \dot{\cup} G^{\mathrm{CG}} \dot{\cup} G^{\mathrm{F}}$ beat players from $M$. There are a few exceptions to this rule, which will be detailed below. 


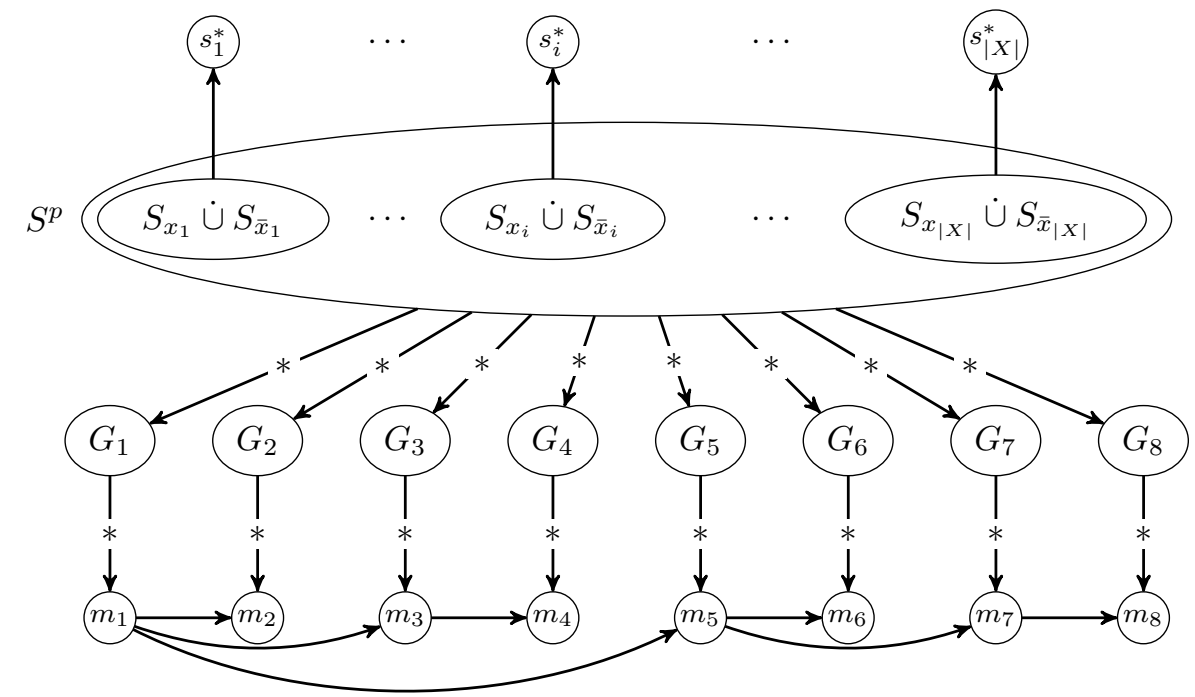

Figure 2: Global structure for the case $k=8$. All arrows not shown in the figure run downwards, horizontal arcs run right to left. Vertices grouped in a component have the same relation with vertices outside the component (unless specified otherwise). The relation of components connected by a starred edge has a few exceptions that are discussed in detail for the individual gadgets: Every element $m_{i}$ beats exactly four of the elements from $G_{i}$ and some $G_{i}$ contain elements that beat some elements from $S^{p}$.

In many places, we will use the right-left-rule, that is elements from sets with a higher index will beat elements from sets with a lower index. For instance, for all $j, j^{\prime} \in[k]$ with $j>j^{\prime}$ and elements $i \in G_{j}, i^{\prime} \in G_{j^{\prime}}$ we have that $i$ beats $i^{\prime}$.

The set $S$ can be partitioned into subsets $S_{j}$ corresponding to each variable of the SAT instance, such that $\forall j \in[|X|]: S_{j}=S_{x_{j}} \dot{\cup} S_{\bar{x}_{j}} \dot{\cup}\left\{s_{j}^{*}\right\}$ where $\left|S_{x_{j}}\right|=$ $\left|S_{\bar{x}_{j}}\right|=3$. We further define the set of particles (these will move between the choice and clause/garbage gadgets) by $S^{p}:=\dot{\bigcup}_{j \in[|X|]}\left(S_{x_{j}} \dot{\cup} S_{\bar{x}_{j}}\right)$. The members of $S^{p}$ follow the right-left-rule between each other, i.e., for $j>j^{\prime}$, elements from $S_{x_{j}}$ and $S_{\bar{x}_{j}}$ beat elements from $S_{x_{j^{\prime}}}$ and $S_{\bar{x}_{j^{\prime}}}$. The players in $\left\{s_{j}^{*} \mid j \in[|X|]\right\}$ follow the right-left-rule amongst themselves. For each $j \in[|X|], s_{j}^{*}$ is beaten by all other members of $S_{j}$ and beats all members of $S^{p} \backslash S_{j}$. The structure is visualized in Figure 2.

The relation of the members of $M$ among themselves is given by the spawning process (see Figure 1).

The only exceptions to the general rule of $S$ beating all other players and $G^{\mathrm{X}} \dot{\cup} G^{\mathrm{CG}} \cup G^{\mathrm{F}}$ beating players from $M$ are listed below, they will be detailed in the description of the individual gadgets.

- Some sets $G_{i}$ contain elements that beat some elements from $S^{p}$.

- Every element $m_{i}$ beats exactly four of the elements from $G_{i}$. 
Lemma 2. For $m_{1}$ to win the tournament, all players $m_{j} \in M$ must reach the fifth round.

Proof. We use induction over the set $M$. The induction will proceed from left to right according to the order specified in the description of the spawning process. First, recall that $\ell_{j}$ denotes the smallest $\ell$ such that $m_{j}$ is present after the $\ell$-th iteration of the spawning process. Further note that for all $j \in[k]$, player $m_{j}$ beats exactly $\left(R-4-\ell_{j}\right)=\left(\log k-\ell_{j}\right)$ players from $M$ that are to his right. The induction hypothesis is as follows.

In order for $m_{1}$ to win the tournament, the following must hold for all $j \in[k]$ :

- If $m_{j}$ was spawned by some player $m_{j^{*}}$, then $m_{j}$ needs to play against $m_{j^{*}}$ in round $\left(R+1-\ell_{j}\right)$.

For the base case, consider $m_{1}$. Player $m_{1}$ was spawned by no other player, hence there is nothing to show.

We now show that for a player $m_{j} \in M$, the induction hypothesis holds, provided that it holds for all players $m_{j^{\prime}} \in M$ with $j^{\prime}<j$. Denote by $m_{j^{*}}$ the player that spawned $m_{j}$ and note that $j^{*}<j$ and hence the induction hypothesis holds for $j^{*}$.

First, note that $m_{j^{*}}$ beats all players $m_{j^{\prime}}$ with $j^{\prime}<j^{*}$ except the one that spawned him. However, by the induction hypothesis they all have to play (and lose) against the player that spawned them, therefore they cannot play against $m_{j^{*}}$ (as they would lose). Thus, the only players that $m_{j^{*}}$ can and is allowed to beat are players from $G_{j^{*}}$ and players $m_{r}$ from $M$ with $r>j^{*}$. He beats exactly four players in $G_{j^{*}}$ and $\left(R-4-\ell_{j^{*}}\right)$ (including $\left.m_{j}\right)$ from $M$ to his right. By the induction hypothesis, $m_{j^{*}}$ needs to reach round $\left(R+1-\ell_{j^{*}}\right)$, he thus has to play against all of these players (including $m_{j}$ ). This proves that $m_{j}$ must play against the player that spawned him.

To see why this has to happen in round $\left(R+1-\ell_{j}\right)$, note that to the left of $m_{j}$ there are $\left(R-4-\ell_{j}\right)$ other players that are spawned by $m_{j^{*}}$. Denote them by $m_{j_{1}^{*}}, m_{j_{2}^{*}}, \ldots, m_{j_{R-4-\ell_{j}}^{*}}$ and note that $R+1-\ell_{j_{i}^{*}}=i+4$. Thus, $m_{j}$ cannot face $m_{j^{*}}$ in round $5,6, \ldots, R-\ell_{j}$ (as other players need to play against $m_{j^{*}}$ in these rounds) and has to proceed at least until round $\left(R+1-\ell_{j}\right)$.

The original claim now follows from the induction hypothesis because $\ell_{j} \leq$ $\log _{2}(k)=\log _{2}\left(\frac{n}{16}\right)=\log _{2}(n)-4=R-4$ and hence $R+1-\ell_{j} \geq R+1-(R-4)=$ 5 .

We now describe the design of the gadgets. These will allow us to conclude that all players from $M$ can reach the fifth round if and only if the 3SAT instance $F$ is satisfiable. Note that all choice gadgets consist of 18 players $\left(\left\{m_{j}\right\} \dot{\cup} G_{j} \dot{\cup} S_{j}\right)$, clause/garbage gadgets consist of 14 players $\left(\left\{m_{j}\right\} \dot{\cup} G_{j}\right)$ and filler gadgets consist of 16 players $\left(\left\{m_{j}\right\} \dot{\cup} G_{j}\right)$. In the first four rounds of a BKT, some players from the choice gadgets thus have to compete in a subtournament against the players from clause/garbage gadgets. The exact way in which this is possible 


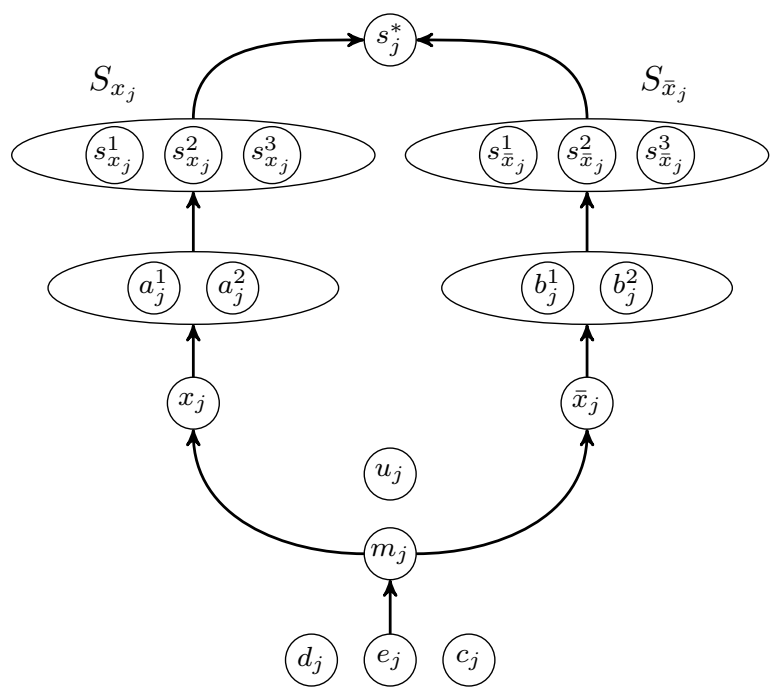

Figure 3: Pairwise comparisons in the $j$-th choice gadget. All arrows not shown in the figure run downwards, horizontal arcs run right to left. Vertices grouped in a component have the same relation with vertices outside the component.

while making sure that $m_{1}$ wins the tournament will be used to encode the 3SAT instance.

Choice gadget. For each $j \in[|X|]$, the $j$-th choice gadget consists of player $m_{j}$, all ten elements of $G_{j}$ and all of $S_{j}$. Note that some elements of $S_{j}$ will appear again in the clause/garbage gadgets. The pairwise comparison graph for these elements (for fixed $j$ ) is shown in Figure 3. The choice gadget is structured in such a way that it is possible for $m_{j}$ to win a subtournament composed of all elements in the gadget except two elements of either $S_{x_{j}}$ or $S_{\bar{x}_{j}}$, as illustrated in Figure 6. We will also show that this is the only way in which $m_{j}$ can reach the fifth round in a tournament won by $m_{1}$.

Clause/garbage gadget. We now describe the internal structure of the clause/garbage gadgets. For each $j \in\{|X|+1,|X|+2, \ldots 2|X|\}$, the $j$-th gadget consists of $m_{j}$ and the 13 elements of $G_{j}$, two of these are denoted $\mathrm{c} / \mathrm{g}$. The pairwise comparison graph for these players is shown in Figure 4. For each clause $c_{i} \in C$ we will call one of the players denoted $c / g$ associated with clause $c_{i}$, all remaining players $c / g$ are garbage players. All players shown in the figure are beaten by all players in $S$ with the following exceptions:

(i) garbage players beat all players from $S^{p}$,

(ii) players associated with clause $c_{i}$ beat all players from the set $S_{x_{j}}$ or $S_{\bar{x}_{j}}$ if $x_{j}$ or $\bar{x}_{j}$ occurs in clause $c_{i}$, respectively. 


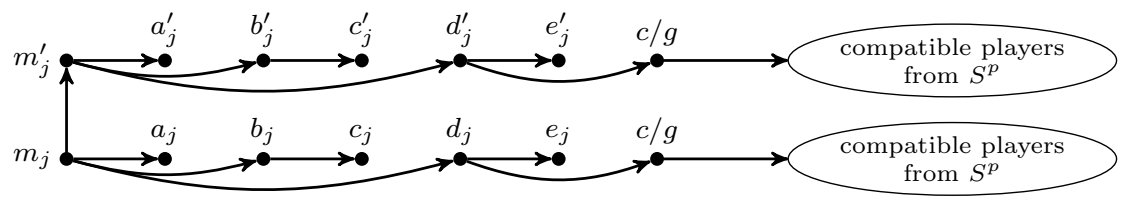

Figure 4: Pairwise comparison graph for a clause/garbage gadget. All arrows not shown in the figure run downwards, horizontal arcs run right to left.

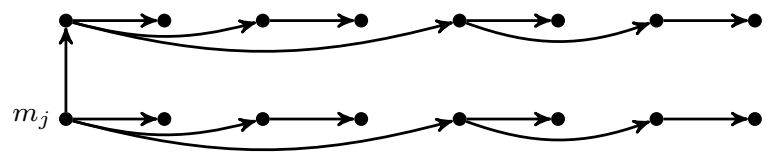

Figure 5: Pairwise comparison graph for a filler gadget. All arrows not shown in the figure run downwards, horizontal arcs run right to left.

The clause gadget is structured in such a way that it is possible for $m_{j}$ to win a subtournament composed of all elements in the gadget with the addition of a compatible element from $S^{p}$ for both $c / g$-players included. We will also show that if we want $m_{1}$ to win the tournament, this is the only way in which $m_{j}$ can reach the fifth round.

Filler gadget. Finally, for each $j \in\{2|X|+1,2|X|+2, \ldots, k\}$, the $j$-th filler gadget consists of $m_{j}$ and 15 players in set $G_{j}$. The pairwise comparison graph relating them to one another is shown in Figure 5, the structure is the same as among the players from $M$.

We note that for all $j \in[k]$, player $m_{j}$ beats exactly four players from outside of the set $M$. This allows us to prove the following lemma.

Lemma 3. For all $j \in[k]$ the following hold:

(i) If all players from $M$ reach the fifth round, then the 16-player subtournament taking $m_{j}$ to the fifth round consists exactly of:

(a) if the $j$-th gadget is a choice gadget: $\left\{m_{j}\right\} \dot{\cup} G_{j} \dot{\cup} S_{j}$ where two elements of either $S_{x_{j}} \dot{\cup}\left\{s_{j}^{*}\right\}$ or of $S_{\bar{x}_{j}} \dot{\cup}\left\{s_{j}^{*}\right\}$ are removed,

(b) if the $j$-th gadget is a clause/garbage gadget: $\left\{m_{j}\right\} \dot{\cup} G_{j}$ and for each of the $c / g$ players in $G_{j}$ one additional element of $S^{p}$ that he can beat,

(c) if the $j$-th gadget is a filler gadget: $\left\{m_{j}\right\} \dot{\cup} G_{j}$.

(ii) If the 16-player subtournament for the first four rounds in which $m_{j}$ is placed consists exactly of (i)a, (i)b, or (i)c for the respective type of the $j$-th gadget, then a draw for the subtournament exists by which $m_{j}$ reaches the fifth round. 
Proof. We prove the lemma by induction over $j$. Assume that the statement holds for all $i<j$ (this also takes care of the case $j=1$ were we do not assume anything).

Note that by induction, all players from $G_{i}, i<j$ have to be seeded in the $i$ th subtournament and are hence no longer available for the $j$-th subtournament. Also, no other player from $M$ may be seeded in the subtournament of $m_{j}$, as otherwise not all players from $M$ can reach the fifth round.

In our analysis we consider positions in the subtournament one after another. We say that a player is no longer available when in order for $m_{j}$ to advance we need that he be placed in one of the positions already considered.

We first consider the case where the $j$-th gadget is a choice gadget. In order to proceed to the fifth round, player $m_{j}$ has to be matched against all four of the players in $G_{j}$ that he can beat. In Figure 3 these are players $\left\{d_{j}, c_{j}, x_{j}, \bar{x}_{j}\right\}$. Of those four players, $d_{j}$ beats no player that is still available. Therefore, $d_{j}$ cannot proceed to the second round and must face $m_{j}$ in the first round. It is therefore no longer available elsewhere. In rounds two to four, $m_{j}$ thus has to be matched against $c_{j}, x_{j}$, and $\bar{x}_{j}$. Of these, $c_{j}$ beats only one player that is still available $\left(e_{j}\right)$ and can therefore only proceed to the second round. By the same argument as before, $m_{j}$ must be matched against $c_{j}$ in the second round, thus $c_{j}$ and $e_{j}$ are no longer available for consideration in later positions. For the third and fourth round we can either match $m_{j}$ first against $x_{j}$ and then against $\bar{x}_{j}$ or the other way around since both those players are capable of reaching the fourth round. Since both $x_{j}$ and $\bar{x}_{j}$ beat exactly three of the available players and $u_{j}$ is among them for both $x_{j}$ and $\bar{x}_{j}$, the player who will be matched against $m_{j}$ in the fourth round must beat $u_{j}$. This match must happen in the first round, as $u_{j}$ beats none of the available players. No matter whether we match $m_{j}$ against $x_{j}$ or $\bar{x}_{j}$ first, $x_{j}$ must beat $a_{j}^{1}$ and $a_{j}^{2}$ whilst $\bar{x}_{j}$ must beat $b_{j}^{1}$ and $b_{j}^{2}$. Depending on which option we chose, $a_{j}^{1}$ and $a_{j}^{2}$ will have to lose either in the second and third round and $b_{j}^{1}$ and $b_{j}^{2}$ in the first and second round or vice versa. Note that, among the players still available, $a_{j}^{1}$ and $a_{j}^{2}$ only beat members of $S_{x_{j}}$ whereas $b_{j}^{1}$ and $b_{j}^{2}$ only beat members of $S_{\bar{x}_{j}}$. This means that if we select $a_{j}^{1}$ and $a_{j}^{2}$ to reach the second and third round, their first- and second-round matches must all be against players from $S_{x_{j}}$ by which all three members of $S_{x_{j}}$ lose against either $a_{j}^{1}$ or $a_{j}^{2}$ in the first or second round. On the other hand, if $a_{j}^{1}$ and $a_{j}^{2}$ only had to get to first and second round, they would only have to play against one member of $S_{x_{j}}$ and the other two could be removed from the subtournament. The same holds for $b_{j}^{1}, b_{j}^{2}$ and $S_{\bar{x}_{j}}$, respectively. This situation is shown in Figure 6 . This proves $(i),(i i)$ is easily verified if one observes that one of the players in $S_{j}^{p}$ that has to make it to the second round can do so by being matched against $s_{j}^{*}$ or a player from $S_{i}^{p}$ with $i<j$.

Now let us consider the case where the $j$-th gadget is a clause gadget, the argument is very similar. Again, player $m_{j}$ has to be matched against all four of the players in $G_{j}$ that he can beat. In Figure 4 these are the players $\left\{a_{j}, b_{j}, d_{j}, m_{j}^{\prime}\right\}$. Of those four players, $a_{j}$ beats no player that is still available. Therefore, $a_{j}$ cannot proceed to the second round and must face $m_{j}$ in the first round. This 


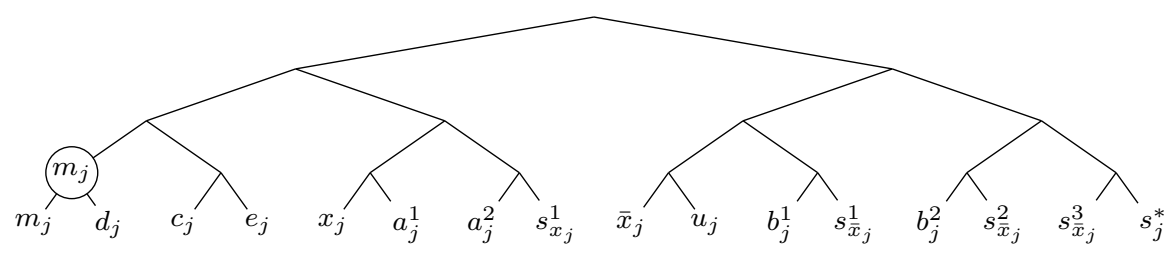

Figure 6: The subtournament for choice gadget $j$. In this case, $x_{j}=$ true is selected.

makes $a_{j}$ unavailable to be used in other positions. In rounds two to four, $m_{j}$ thus has to be matched against $b_{j}, d_{j}$, and $m_{j}^{\prime}$. Of these, $b_{j}$ beats only one player that is still available $\left(c_{j}\right)$ and can therefore only proceed to the second round. The same argument as before forces $m_{j}$ to be matched against $b_{j}$ in the second round removing $b_{j}$ and $c_{j}$ from availability. Player $m_{j}$ now beats $d_{j}$ and $m_{j}^{\prime}$ amongst the available players. Player $d_{j}$ only beats two other players, therefore $m_{j}$ will beat him in the third round. Player $d_{j}$ beats $e_{j}$ and a $c / g$ player. Player $e_{j}$ beats none of the available players, therefore $d_{j}$ must beat him in the first round. This makes $e_{j}$ unavailable. The $c / g$ player beats compatible players from $S^{p}$ and can therefore go to the second round if one of those is available. Player $m_{j}$ is left with only one player to beat to get to the fifth round which is $m_{j}^{\prime}$. Player $m_{j}^{\prime}$ can and needs to reach the fourth round. The sub-tournament enabling $m_{j}^{\prime}$ to reach the fourth round is structured exactly the same way as the one just described. Therefore $m_{j}$ can go to the fifth round if and only if we can add to the subtournament consisting of $m_{j}$ and $G_{j}$ one compatible (beatable) player from $S^{p}$ for each $c / g$ player, which proves both $(i)$ and (ii).

The filler gadget is built by the spawning process. If the $j$-th gadget is a filler gadget, this guarantees that $m_{j}$ beats only four players, one of which beats no one in the gadget. $m_{j}$ therefore needs to beat him to get to the second round and therefore that player is no longer available. The spawning process also guarantees there is a second player that beats only one other available player in the gadget and can only get to the second round. $m_{j}$ must beat him in the second round. We can continue using the same argument until the fifth round to show that $m_{j}$ can go to the fifth round if and only if all players from $G_{j}$ are placed in the subtournament together with him, which proves both $(i)$ and $(i i)$.

Note that in light of Lemma 2, it follows by induction from Lemma 3 that $m_{1}$ can win the tournament if and only if the composition of all 16-player subtournaments covering the first four rounds is exactly as prescribed. We can now finally prove the following lemma.

Lemma 4. Let $F=(X, C)$ be a 3SAT instance where each literal appears at most twice. There exists a set of players $N$ with pairwise comparison matrix $P$ and a distinguished player $m_{1} \in N$ such that $F$ is satisfiable if and only if we can find a draw $\sigma$ such that $m_{1}$ wins the tournament $\mathrm{T}(N, \sigma)$.

Proof. Let $N, m_{1}$ and $P$ be as specified above. First, note that by Lemma 1 and Lemma 2, $m_{1}$ can win the tournament if and only if all players from the 
set $M$ reach the fifth round. This is possible if and only if each player $m_{j} \in M$ can win a 16-player subtournament that consists exactly of the players stated in Lemma 3. We thus have to show that a draw in which each player $m_{j} \in M$ wins a 16-player subtournament with exactly the players stated in Lemma 3 exists if and only if the 3SAT instance is satisfiable.

$(\Leftarrow)$ Let the formula $F$ be satisfied by a truth assignment $A$. We construct a BKT over $N$ in which $m_{1}$ wins. For all $j$ where the $j$-th gadget is a choice gadget, we place all players from $\left\{m_{j}\right\} \cup G_{j} \cup \dot{\cup} S_{j}$ with the exception of two players from $S_{x_{j}}$ (if $A\left(x_{j}\right)=$ true) or $S_{\bar{x}_{j}}$ (if $A\left(x_{j}\right)=$ false) in one subtournament for the first four rounds. These are exactly 16 players and by Lemma 3(ii), we can choose the draw in such a way that $m_{j}$ wins the subtournament.

Note that the choice gadgets use all players from $S$ except for two players of each set $S_{x_{j}}$ (or $S_{\bar{x}_{j}}$ ) where $x_{j}$ (or $\bar{x}_{j}$ ) is a literal that evaluates to true. In other words, for every literal that evaluates to true, two players remain that can be beaten by every player associated with a clause in which the literal players appear.

For the clause/garbage gadgets, to use Lemma 3b) we need for each $c / g$ player one additional player that he beats. If $c / g$ is associated with a clause $c_{i} \in C$, pick one of the literals in $c_{i}$ that evaluates to true, say $\bar{x}_{j}$, and assign one of the two players from $S_{\bar{x}_{j}}$ mentioned above to this $c / g$ player. Note that at most two such players are required for any literal, as every literal only appears twice. To all garbage players, assign an arbitrary remaining player from set $S$. Note that, after composing the subtournaments for all choice gadgets, $2|X|$ players from the set $S$ were left. As there are $2|X| c / g$ players, we can assign a player from $S$ to each of these.

For all $j$ where the $j$-th gadget is a clause/garbage gadget, we now place all players from $\left\{m_{j}\right\} \dot{\cup} G_{j}$ in one subtournament for the first four rounds, together with the two players that are assigned to the $c / g$ players in $G_{j}$. By Lemma 3(ii), we can chose a draw such that $m_{j}$ wins this subtournament.

For all $j$ where the $j$-th gadget is a filler gadget, we place all players from $\left\{m_{j}\right\} \dot{\cup} G_{j}$ in one tournament for which, by Lemma 3(ii), we can find a draw where $m_{j}$ wins.

$(\Rightarrow)$ Let a draw be given such that all players in $M$ win a 16-player subtournament consisting exactly of the players stated in Lemma 3. First, note that whenever a player $s_{j}^{*}$ is not placed in the $j$-th choice gadget, we get a contradiction as by Lemma 3, this player cannot be placed anywhere else while all players from $M$ still reach the fifth round. The following truth assignment is thus well-defined: $A\left(x_{j}\right)=$ true if $m_{j}$ 's subtournament includes all players from $S_{j}$ except two players from $S_{x_{j}}, A\left(x_{j}\right)=$ false if $m_{j}$ 's subtournament includes all players from $S_{j}$ except two players from $S_{\bar{x}_{j}}$.

We now show that $A$ satisfies all clauses. Let $c_{i} \in C$ be a clause and $c / g$ the player associated with the corresponding clause gadget. Let $j^{*}$ be the index of that gadget. By the assumption, $m_{j^{*}}$ wins the corresponding subtournament, thus Lemma 3 implies that it contains a player that $c / g$ beats, i.e. a player from set $S_{x_{j}}$ (or $S_{\bar{x}_{j}}$ ) where $x_{j}$ (or $\bar{x}_{j}$ ) occurs in clause $C_{i}$. By the definition of $A$, that player can only be used in the subtournament if the corresponding literal 
evaluates to true (as otherwise, the player is used in $m_{j}$ 's subtournament).

This concludes the proof of Theorem 1.

The following Lemma will be required for Theorem 6 . We prove it here, as it can be used to show that it remains computationally hard to decide whether a particular player can win a BKT, even if we already know that that player is a particularly strong player: A player $a$ is called a king if for every player $b$ that $a$ does not beat, there exists a third player $c$ such that $a$ beats $c$ and $c$ beats $b$. Using the next lemma, we will show that TFP remains NP-hard if restricted to instances where $m_{1}$ is a king.

A related theorem has previously been proved by Kim and Vassilevska Williams [17] using our Theorem 1 . The result from [17] is slightly more general in that it requires $m_{1}$ to be a particularly strong king (one that directly beats $1 / 4$ of all players). Leaving out this additional requirement, we can make a simpler argument and only require an instance half the size.

Lemma 5. Let $k \in \mathbb{N}$ and $N^{\prime}$ a set of players with $\left|N^{\prime}\right|=2^{k}$ and a distinguished player $m_{1} \in N^{\prime}$.

(i) Let $Z$ be another (disjoint) player set with $|Z|=\left|N^{\prime}\right|$ and $z_{1} \in Z$ a player from $Z$. Let $P$ be a pairwise comparison matrix on $N=N^{\prime} \dot{\cup} Z$ such that

- $z_{1}$ beats $z$ for any $z \in Z \backslash\left\{z_{1}\right\}$

- $z$ beats $p$ for any pair $(z, p) \in\left(Z \times N^{\prime}\right) \backslash\left\{\left(z_{1}, m_{1}\right)\right\}$

- $m_{1}$ beats $z_{1}$ (see Figure 7).

Then, there is a draw $\sigma$ of $N$ such that $m_{1}$ wins the tournament $\mathrm{T}(N, \sigma)$ if and only if there is a draw $\sigma^{\prime}$ of $N^{\prime}$ such that $m_{1}$ wins the tournament $\mathrm{T}\left(N^{\prime}, \sigma^{\prime}\right)$.

(ii) Let $P^{\prime}$ be a pairwise comparison matrix on $N^{\prime}$. There exists a set of players $Z$ and pairwise comparison matrix $P$ on $N^{\prime} \dot{\cup} Z$ satisfying the conditions from (i) such that $P$ coincides with $P^{\prime}$ on $N^{\prime}$.

Proof. For part (i), we prove both directions separately.

$(\Rightarrow)$ Assume that there exists a draw $\sigma^{\prime}$ such that $m_{1}$ wins the tournament $\mathrm{T}\left(N^{\prime}, \sigma^{\prime}\right)$. Construct $\sigma$ in such a way that the final is a match between the winners of two sub-tournaments $A$ and $B$ where in $A$, all players from $N^{\prime}$ are seeded according to $\sigma^{\prime}$ and in $B$ all players from $Z$ are seeded in an arbitrary way. The sub-tournament $B$ is won by $z_{1}$ (as it beats all other players that compete in $B$ ) and sub-tournament $A$ is won by $m_{1}$ (by Lemma 4 as $A$ is exactly the tournament $\left.\mathrm{T}\left(N, \sigma^{\prime}\right)\right)$. In the final, $m_{1}$ wins against $z_{1}$ and hence $m_{1}$ wins the tournament $\mathrm{T}(N, \sigma) .(\Leftarrow)$ Assume that there is no draw $\sigma^{\prime}$ such that $m_{1}$ wins the tournament $\mathrm{T}\left(N^{\prime}, \sigma^{\prime}\right)$. Again, let $\sigma$ be a draw for $N$ such that the final is a match between the winners of two sub-tournaments $A$ and $B$ and assume w.l.o.g. that $m_{1}$ is seeded in $A$. We differentiate three cases: 


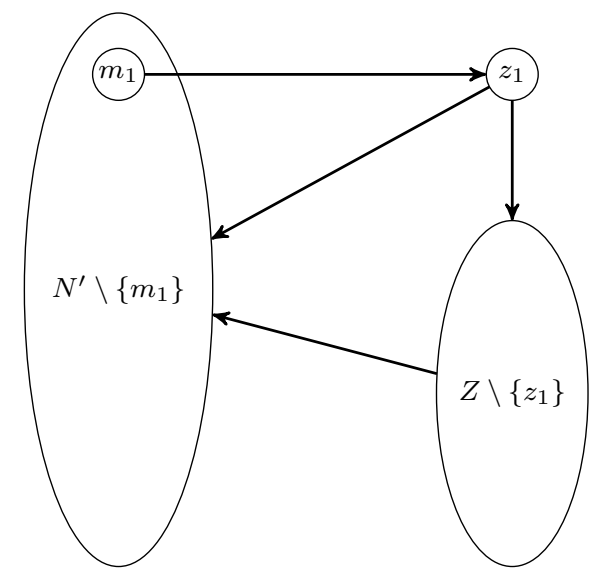

Figure 7: Pairwise comparison among members of the set $N$ from Lemma 5.

(i) If $z_{1}$ is also seeded in $A$, then there is at least one player from $Z \backslash\left\{z_{1}\right\}$ that is seeded in $B$ (because only $\left|N^{\prime}\right|=|Z|$ players can compete in $A$ ). Hence a player $z \in Z \backslash\left\{z_{1}\right\}$ will win the sub-tournament $B$ and even if $m_{1}$ wins $A$, he will be beaten by $z$ in the final.

(ii) If $z_{1}$ is not seeded in $A$ but some other players from $Z$ are, then one of them will win the tournament $A$ and hence $m_{1}$ cannot win.

(iii) If no player from $Z$ is seeded in $A$, then by the assumption there is no draw such that $m_{1}$ can win the sub-tournament $A$.

Hence, there is no draw $\sigma$ such that $m_{1}$ wins the tournament $\mathrm{T}(N, \sigma)$ if $m_{1}$ cannot win the tournament among the players in $N^{\prime}$.

For part (ii) of Lemma 5, a suitable instance can easily be constructed, as no assumptions are made on the structure of $P$ restricted to $N^{\prime}$.

Theorem 2. TFP is NP-complete even if the distinguished player is a king.

Proof. First, note that in any instance satisfying the conditions of Lemma 5, $m_{1}$ is a king (by beating $z_{1}$ who in turn beats every other player).

Let $F=(X, C)$ be a 3SAT instance where each literal appears at most twice. By Lemma 4, we can find a player set $N^{\prime}$, pairwise comparison matrix $P^{\prime}$ and distinguished player $m_{1}$ such that $F$ is satisfiable if and only there is a draw $\sigma$ such that $m_{1}$ can win $T\left(N^{\prime}, \sigma\right)$.

By Lemma 5(ii), we can obtain a suitable instance such that $m_{1}$ is a king and the statement follows from Lemma 5(i).

\subsection{Implications of Theorem 1}

Theorem 1 simultaneously implies a number of results from the literature, in particular Theorem 1, 2, and 3 in Vu et al. [34] and Theorem 1 in Vassilevska Williams [32]. It also yields some further corollaries. For PTFP and $\alpha \geq 1$, 
an algorithm is called an $\alpha$-approximation algorithm for the maximum winning probability if it can find a draw in which the winning probability of the given player $i$ is at least $\operatorname{mwp}(i, N, P) / \alpha$.

Corollary 1. Unless $P=N P$, there exists no deterministic polynomial-time algorithm for PTFP that approximates the maximum winning probability of a player within any given positive factor.

Proof. The approximation algorithm can be used to check in polynomial time whether the maximum winning probability is zero or non-zero. If the algorithm is deterministic, it can hence solve TFP in polynomial time.

It immediately follows from Theorem 1 that \#TFP is NP-hard. We next highlight that even randomisation is not very helpful for \#TFP. Let $\Gamma$ be a finite alphabet in which we agree to describe our problem instances and solutions. A fully polynomial time randomized approximation scheme (FPRAS) for a function $f: \Gamma^{*} \rightarrow \mathbb{Q}$ is an algorithm that takes input $x \in \Gamma^{*}$ and a parameter $\epsilon \in \mathbb{Q}_{>0}$, and returns with probability at least $\frac{3}{4}$ a number between $f(x) /(1+\epsilon)$ and $(1+\epsilon) f(x)$. Moreover, an FPRAS runs in time polynomial in the size of $x$ and $1 / \epsilon$. RP is the complexity class consisting of problems that can be solved in randomized polynomial time. The statement below follows from Proposition 8 in Welsh and Merino [37] and Theorem 1.

Corollary 2. Unless $N P=R P$, there is no FPRAS for \#TFP.

Proof. The statement follows from Theorem 1 and the well-known fact (see for e.g., Proposition 8 in [37]) that if $f: \Gamma^{*} \longrightarrow \mathbb{N}$ is such that deciding whether $f$ is non-zero or not is NP-hard, then there is no FPRAS for $f$ unless NP $=\mathrm{RP}$.

Hence, unless NP $=\mathrm{RP}$, there also does not exist an FPRAS for computing the probability of a player winning a BKT for a draw chosen uniformly at random.

\section{Tractable Cases}

We first examine a natural case in which players are divided into player types: the outcome of any match is determined only by the type of the respective players. In other words, given two types $i$ and $j$, it is either the case that all players from $i$ win against all players from $j$ or the other way round. This is an interesting case as in sports tournaments it may be that many players are of the same or similar quality, i.e., of the same type. The result of matches between players of the same type is irrelevant as we do not care which player in each type wins. In this variant that we call \#TFP-types, the objective is to count the number of draws for which a player of a given type wins. We first adapt the

concept of the pairwise comparison matrix. For two player types $i$ and $j$, define $P_{i j}=1$ if an $i$-player wins a match between $i$ and $j$ and $P_{i j}=0$ otherwise. Note that this definition is chosen such that $P_{i i}=1$ for all player types $i$. 
Theorem 3. \#TFP-types is polynomial-time solvable if there are a constant number of player types.

Proof. We will use a dynamic programming scheme. All vectorial inequalities assume equal dimensions and are meant component-wise. Let $k$ be the number of different player types. We denote the number of players of type $i$ by $\eta_{i}$. Let $N_{x}:=\left\{\eta=\left(\eta_{1}, \ldots, \eta_{k}\right) \geq 0 \mid \eta_{1}+\cdots+\eta_{k}=2^{x}\right\}$ and denote by \#TFP $(i, x, \eta \in$ $N_{x}$ ) the number of draws for an $x$-round tournament involving $\eta_{j}$ players of type $j$ for all $j \in[k]$ in which a player of type $i$ wins the tournament. We will assume that the players of one type are not distinguishable. For fixed $i$ and $x$, there are $\left(\begin{array}{c}k+2^{x}-1 \\ k-1\end{array}\right)$ such problems that potentially need to be considered. This is because for positive integers $p, q$ there are $\left(\begin{array}{c}p+q-1 \\ q-1\end{array}\right)$ weak compositions of $p$ into $q$ parts, i.e., there are $\left(\begin{array}{c}p+q-1 \\ q-1\end{array}\right)$ distinct ways to write a sum of $q$ non-negative integers that evaluates to $p$. The number \# $\operatorname{TFP}(i, x, \eta)$ is computed via the following recursion.

$$
\begin{aligned}
& \# \operatorname{TFP}(i, x, \eta)= \\
& \qquad \sum_{\substack{\eta^{\prime} \in N_{x-1} \\
\eta^{\prime} \leq \eta}}\left(\# \operatorname{TFP}\left(i, x-1, \eta^{\prime}\right) \cdot \sum_{\substack{j \in[k] \\
P_{i j}=1 \\
\eta_{j}^{\prime}<\eta_{j}}} \# \operatorname{TFP}\left(j, x-1, \eta-\eta^{\prime}\right)\right)
\end{aligned}
$$

The recursion considers all possible opponents of a player of type $i$ at round $x$, as well as all possibilities for the $(x-1)$-round seedings of the subtournaments that the players of type $i$ and $j$ need to win in order to reach the final round.

The base cases are given by

$$
\# \operatorname{TFP}(i, 0, \eta)= \begin{cases}1 & \eta_{i}=1 \text { and } \forall j \neq i: \eta_{j}=0 \\ 0 & \text { otherwise }\end{cases}
$$

for all $i \in[k]$. Eq. (3) only uses values of \#TFP $\left(j, y, \eta^{\prime}\right)$ with $y<x$, thus every problem only needs to be solved once. For constant $k$, solving a problem requires $O\left(n^{k}\right)$ operations and for constant $k$ it holds that $\left(\begin{array}{c}k+2^{x}-1 \\ k-1\end{array}\right) \in O\left(n^{k}\right)$. Thus, $O\left(\log (n) n^{2 k}\right)$ operations are necessary to compute $\# \operatorname{TFP}\left(i, \log (n),\left(n_{1}, \ldots, n_{k}\right)\right)$.

The second tractable case that we identify is the case when the players can be linearly ordered by strength. This is a common rule of thumb used when guessing who will win a tournament and is supported by empirical results in the literature [24].

Theorem 4. \#TFP is polynomial-time solvable if there is a linear ordering of strengths of the players with at most a constant number of pairwise relations flipped.

Proof. Let $b$ be the number of backwards arcs that do not respect the linear ordering. We can show by induction that the problem can be reduced to \#TFPtypes with at most $4 b+3$ player types. For $b=0$, we simply divide the players 


\begin{tabular}{ccc}
$y$ & time $T(n)$ & space $S(n)$ \\
\hline 2 & $O\left(2.8285^{n}\right)$ & $O\left(1.7548^{n}\right)$ \\
3 & $O\left(3.3636^{n}\right)$ & $O\left(1.4576^{n}\right)$ \\
4 & $O\left(3.6681^{n}\right)$ & $O\left(1.2634^{n}\right)$ \\
$\log n$ & $4^{n+o(n)}$ & $\operatorname{poly}(n)$
\end{tabular}

Table 1: Running times and space requirements for the algorithm of Theorem 5 for various time-space trade-offs.

into the 3 types $N^{-}\left(i^{*}\right),\left\{i^{*}\right\}$, and $N^{+}\left(i^{*}\right)$, where $N^{-}\left(i^{*}\right)$ and $N^{+}\left(i^{*}\right)$ are the set of players that beat $i^{*}$ and are beaten by $i^{*}$, respectively. By induction, assume the statement holds for all $b^{\prime}<b$. To show that it holds for $b$ backwards arcs, select an arbitrary backwards arc $a=(u, v)$, reverse it, and group the players into $4(b-1)+3$ player types. We partition the players of $u$ 's type into three new types: $\{u\}$, those players that beat $u$, and those that are beaten by $u$. We do the same for $v$, and now we can reverse the arc $a$ again, as it originally was. Thus we have $4(b-1)+3+4$ player types for the instance. Since the number of player types is constant, the theorem follows from Theorem 3 .

\section{An Exponential Time Algorithm for \#TFP}

\#TFP can be trivially solved in time $2^{O(n \log n)}$ via a brute-force enumeration of all possible draws. In exponential time algorithmics [9], the aim is to design algorithms solving the problem exactly with worst-case running times outperforming the brute-force solution. In this section we give an algorithm for \#TFP running in time $O\left(2.8285^{n}\right)$ using space $O\left(1.7548^{n}\right)$. If only polynomial space is available, the running time becomes $4^{n+o(n)}$, and we give a range of possible time-space trade-offs. After the publication of our conference paper, Kim and Vassilevska Williams [17] improved our running time to $O\left(2^{n}\right.$ poly $\left.(n)\right)$ using $O\left(2^{n}\right.$ poly $\left.(n)\right)$ space. However, our algorithm also works if less space is available. The algorithm is based on the recursion formula (3) and memoization used at various levels of the recursion. We use poly $(n)$ to stand for a polynomial function in $n$.

Theorem 5. For every $y, 2 \leq y \leq \log n$, there is an algorithm solving \#TFP in time $T(n)=\operatorname{poly}(n) \cdot \prod_{p=0}^{y-1} \frac{n}{2^{p}} \cdot 2^{n / 2^{p}}$ and space $S(n)=\operatorname{poly}(n) \cdot\left(2^{y}\right)^{n / 2^{y}}$. $\left(\frac{2^{y}}{2^{y}-1}\right)^{n-n / 2^{y}}$.

Proof. We start with the polynomial space algorithm. The algorithm recursively evaluates the formula (3) for the special case where each player type consists of one player. We say that player $i$ corresponds to player type $\eta_{i}$. The evaluation is top-down, i.e., to evaluate $\# \operatorname{TFP}(i, x, \eta)$ the algorithm performs $O\left(\left|N_{x}\right| \cdot 2^{\left|N_{x}\right|}\right)$ recursive calls to itself solving for each $\eta^{\prime} \in N_{x-1}$ where $\eta^{\prime} \leq \eta$ the subinstance $\# \operatorname{TFP}\left(i, x-1, \eta^{\prime}\right)$ and the subinstances $\# \operatorname{TFP}\left(j, x-1, \eta-\eta^{\prime}\right)$ for all $j \in[n]$ such that $P_{i j}=1$ and $\eta_{j}-\eta_{j}^{\prime}=1$. The base cases are as in the proof of Theorem 3, and the algorithm returns $\# \operatorname{TFP}(i, \log n,(1, \ldots, 1))$. 
For $n$ players, the number of recursive calls of the algorithm is upper bounded by $R(n) \leq n \cdot 2^{n} \cdot R\left(\frac{n}{2}\right)$, which evaluates to $R(n)=\prod_{p=0}^{\log n} \frac{n}{2^{p}} \cdot 2^{n / 2^{p}}=4^{n+o(n)}$. The running time $T(n)$ of the algorithm is within a polynomial factor of $n$ of the number of recursive calls.

Using memoization, we can avoid repeating calculations at the expense of exponential space usage. The algorithm uses a table indexed by players, level, and player type vector. We say that we use memoization at level $x$ if, when evaluating $\# \operatorname{TFP}\left(j, x^{\prime}, \eta\right)$ with $x^{\prime} \leq x$, the algorithms first checks in this table whether this recursive call has already been evaluated. Only if the value has not yet been computed, it computes the result recursively, and stores it in the table. Then, it returns the result that is stored in the table. To evaluate \#TFP $\left(j, x^{\prime}, \eta\right)$ with $x^{\prime}>x$, it does not use the table, and computes it recursively as in the polynomial space algorithm.

At level $\log n$, only one evaluation is made. At level $(\log n)-1, O\left(n 2^{n}\right)$ evaluations are made, but none of them is evaluated more than once. Therefore, we will use memoization at level $(\log n)-y$, where $y$ varies from 2 to $\log n$. The number of table entries used by memoization is upper bounded by $n$ times the number of subsets of size at most $n / 2^{y}$. Using Stirling's approximation for factorials, and a binary representation of integers from 0 to $n$ ! using $O(n \log n)$ bits [?], the space usage of the algorithm is upper bounded by $S(n)=\operatorname{poly}(n)$. $\left(2^{y}\right)^{n / 2^{y}} \cdot\left(\frac{2^{y}}{2^{y}-1}\right)^{n-n / 2^{y}}$. The running time of the algorithm is the time used for the recursion without memoization, $\operatorname{poly}(n) \cdot \Pi_{p=0}^{y-1} \frac{n}{2^{p}} \cdot 2^{n / 2^{p}}$, plus the time for the part with memoization, which is upper bounded by $S(n) \cdot n \cdot 2^{n / 4}=O\left(2.0868^{n}\right)$, since at most $S(n)=O\left(1.7548^{n}\right)$ entries are computed and each computation retrieves $O\left(n \cdot 2^{n / 4}\right)$ table values. Thus, for any $y, 2 \leq y \leq \log n$, we obtain an algorithm with running time $T(n)=\operatorname{poly}(n) \cdot \Pi_{p=0}^{y-1} \frac{n}{2^{p}} \cdot 2^{n / 2^{p}}$ using space $S(n)=\operatorname{poly}(n) \cdot\left(2^{y}\right)^{n / 2^{y}} \cdot\left(\frac{2^{y}}{2^{y}-1}\right)^{n-n / 2^{y}}$

Various time and space requirements of the algorithm are reported in Table 1. Using the rule of thumb that for current computing architectures, the space requirements of an algorithm become a bottleneck if they exceed the square root of the time requirements, the analyses for $y=2$ and $y=3$ currently seem the most relevant.

\section{Double-Elimination Tournaments}

In double-elimination tournaments there are two brackets: the winners bracket where the competition proceeds exactly as in a BKT and the losers bracket where the losers from the BKT are mapped into a new knockout tournament. After (or concurrently) with the conclusion of the winners bracket, the losers bracket tournament is run. The champion of the losers bracket plays (possibly again) the champion of the winners bracket (the BKT winner); in a deterministic setting the winner of this final match is the winner of the overall 
tournament. Just like BKTs, we can define TFP for double-elimination tournaments. Stanton and Vassilevska Williams [30] state the complexity of the TFP for double-elimination tournaments as an open problem. We show that the problem is NP-complete.

Double elimination tournaments $[8,14]$ have been present in sports for decades, and were mathematically defined by Stanton and Vassilevska Williams [30]. In the definition by Stanton and Vassilevska Williams [30] a double elimination tournament is defined by the shape of the losers bracket and a link function that maps losers from the BKT into the losers bracket. This function maps matches in the BKT to leaves of the losers bracket. Stanton and Vassilevska Williams [30] also develop several desiderata by which the losers bracket can be evaluated including: balance, that the losers bracket should be a balanced tournament; round-fairness, that losers should compete against other losers who lost in (nearly) the same round; and repeat-avoidance, link functions should minimize the possibility of two teams meeting both in the winners and losers bracket.

Consider the 16 entrant example of a winners bracket, (implicit) link function, and corresponding losers bracket illustrated in Figure8. Our winners bracket consists of two maximal sub-tournaments, one starting below the circled $m_{1}$ node and one below the circled $z_{1}$ node which we will call $N^{\prime}$ and $Z$ following the convention from Lemma 5. The depicted losers bracket is both round-fair and balanced according to the definitions given by Stanton and Vassilevska Williams [30].

Imagine that the tournament graph of the players $\left\{m_{1}, \ldots, m_{8}, z_{1}, \ldots, z_{8}\right\}$ is generated using the pairwise relation in Lemma 5 with the additional constraint that lower number elements of $Z$ defeat higher number elements of $Z$. Hence, in the winners bracket the left hand player of every leaf pairing would advance, as illustrated in Figure 8. To construct our losers bracket, we use the fair, balanced bracket described by Stanton and Vassilevska Williams [30].

We can extend the construction depicted in Figure 8 to BKTs with any number of players. Note also that we can use any conceivable loser bracket setup and link function, in particular the following theorem als holds for double elimination tournaments with balanced losers brackets and round-fair link functions.

Theorem 6. The tournament fixing problem is NP-complete for double elimination tournaments, regardless of any restrictions on losers bracket setup and link function.

Proof. In Lemma 5 we define the conditions on two sub-tournaments of equal size, $N^{\prime}$ and $Z$, where $N^{\prime}$ corresponds to a TFP instance. By Lemma 5, within the $\mathrm{Z}$ bracket $z_{1}$ beats everyone and everyone in $Z$ beats everyone in $N^{\prime}$ with the exception that $m_{1}$ defeats $z_{1}$. We will show that $m_{1}$ can win the double elimination tournament if and only if we can find a solution to the TFP instance defined on $N^{\prime}$.

Formally, let $F=(X, C)$ be a 3SAT instance where each literal appears at most twice. By Lemma 4 we can find a player set $N^{\prime}$, pairwise comparison matrix $P^{\prime}$ and distinguished player $m_{1}$ such that $F$ is satisfiable if and only if there is 


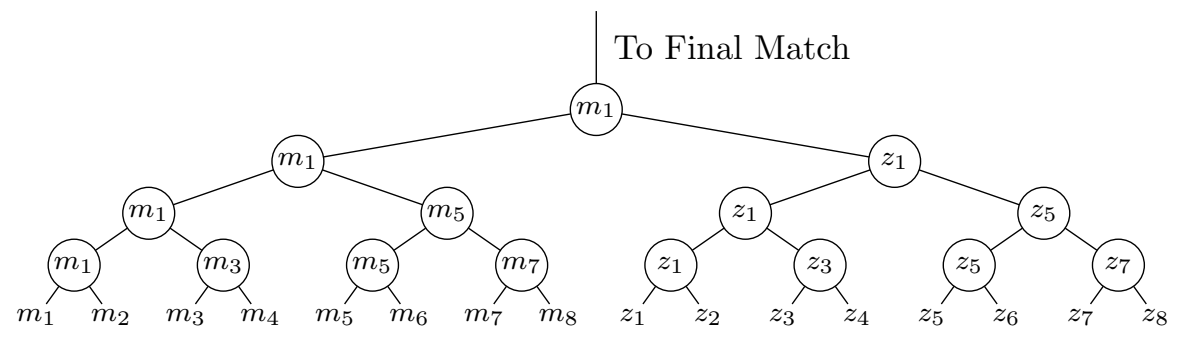

Winners Bracket

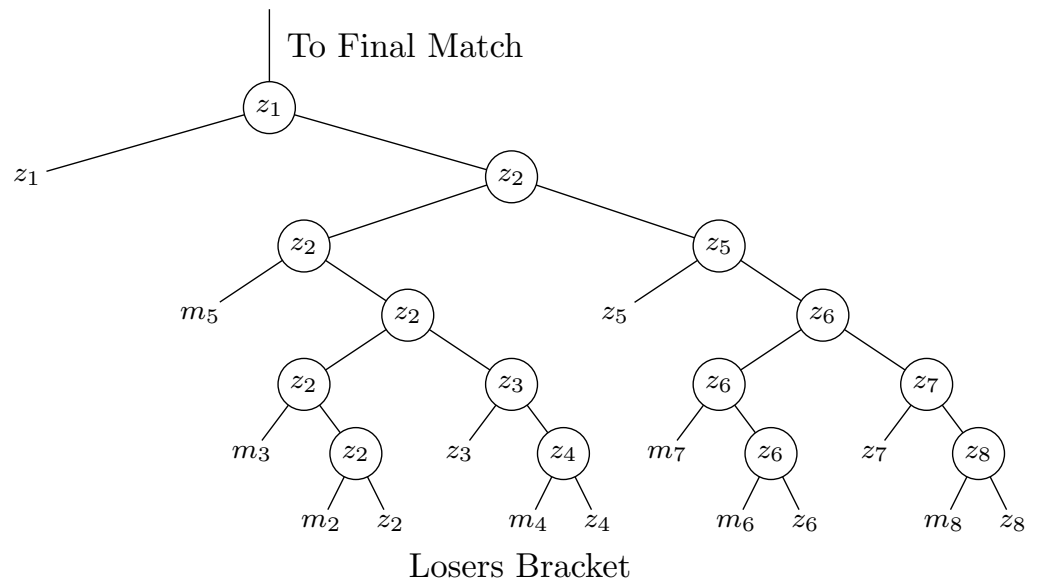

Figure 8: Example of a winners bracket (above) and losers bracket (below) where each left hand player wins each round in their respective $(m$ and $z$ ) maximal sub-tournaments. The champion of the losers bracket plays the champion of the winners bracket in a final match. 
a draw $\sigma^{\prime}$ such that $m_{1}$ can win $\mathrm{T}\left(N^{\prime}, \sigma^{\prime}\right)$. Using Lemma 5(ii), we can extend the instance to the set of players $N:=N^{\prime} \dot{\cup} Z$ with pairwise comparison matrix $P$ that satisfies the properties defined in Lemma 5 part (i). By Lemma $5(\mathrm{i}), F$ is satisfiable if and only if there is a draw $\sigma$ such that $m_{1}$ can win $\mathrm{T}(N, \sigma)$. It remains to show that there is a draw $\sigma$ such that $m_{1}$ can win $\mathrm{T}(N, \sigma)$ if and only if there is a draw $\sigma^{*}$ such that $m_{1}$ wins the double elimination tournament.

$(\Rightarrow)$ We show that if $m_{1}$ wins $\mathrm{T}(N, \sigma)$ then he wins the double elimination tournament by the same draw $\sigma^{*}=\sigma$.

By Lemma 5 , the winners' bracket is won by $m_{1}$. This means that at some point, player $z_{1}$ is eliminated from the winners' bracket and enters the losers' bracket. As $z_{1}$ beats all players except $m_{1}$ (and hence in particular all players in the losers' bracket), $z_{1}$ wins the losers' bracket and faces $m_{1}$ in the final, where he loses and $m_{1}$ wins the double elimination tournament.

$(\Leftarrow)$ Suppose that $m_{1}$ wins the double elimination tournament by some draw $\sigma^{*}$. If $m_{1}$ proceeds to the final as winner of the winner's bracket (which is a BKT of $N)$, then he also wins $\mathrm{T}\left(N, \sigma^{*}\right)$.

Suppose therefore that by the draw $\sigma^{*}$, player $m_{1}$ is at some point eliminated from the winners' bracket. This means that the winners' bracket will be won by some player from $Z$. We show that $m_{1}$ cannot win the double elimination tournament in this case.

If $m_{1}$ does not win the winners' bracket, then $z_{1}$ must win the winners' bracket for $m_{1}$ to win the tournament, since $m_{1}$ is beaten by all other players from $Z$. Therefore, $z_{1}$ will never enter the losers' bracket. This means that some player from $Z \backslash z_{1}$ will win the losers' bracket, as every player from $Z \backslash z_{1}$ beats all other players (those from $N^{\prime}$, including $m_{1}$ ) that enter the losers' bracket at some point. Hence, $m_{1}$ cannot win the losers' bracket and therefore cannot win the tournament.

This proves that $m_{1}$ cannot win the double elimination tournament as winner of the losers' bracket. Therefore, $m_{1}$ can only win the double elimination tournament resulting from the draw $\sigma^{*}$ by advancing through the winner's bracket. This means that he wins $\mathrm{T}\left(N, \sigma^{*}\right)$ which concludes the only-if part of the proof.

As noted before Lemma 5, a similar argument can be constructed using the result from [17]. It follows from Theorem 6 , that the probabilistic generalization of the problem is NP-hard as well. Similarly, we get similar corollaries as Corollaries 1 and 2 for double elimination tournaments.

\section{Conclusions}

In this paper we considered problems related to tournament fixing. Although being able to change draws is not always realistic, the computational problems that are considered have been analyzed in post analysis of tournament draws and also shed light on the relative strengths of players. Our main result is that TFP is NP-complete. We discussed a number of implications of the result. We complement the computational hardness result in the paper by presenting 
algorithms for \#TFP — both for the general case as well as restricted cases. A possible future direction is to propose parameterized algorithms for TFP.

\section{Acknowledgments}

Haris Aziz is supported by a Julius Career Award. Serge Gaspers is the recipient of an Australian Research Council (ARC) Future Fellowship (FT140100048) and acknowledges support under the ARC's Discovery Projects funding scheme (DP150101134).

\section{References}

[1] Aronshtam, L., Cohen, H., Shrot, T., 2017. Tennis manipulation: Can we help Serena Williams win another tournament? Annals of Mathematics and Artificial Intelligence 80 (2), 153-169.

[2] Aziz, H., Brill, M., Fischer, F., Harrenstein, P., Lang, J., Seedig, H. G., 2012. Possible and necessary winners of partial tournaments. In: Proceedings of the 11th International Conference on Autonomous Agents and Multiagent Systems (AAMAS). IFAAMAS, pp. 585-592.

[3] Aziz, H., Gaspers, S., Mackenzie, S., Mattei, N., Stursberg, P., Walsh, T., 2014. Fixing a balanced knockout tournament. In: Proceedings of the 28th AAAI Conference on Artificial Intelligence (AAAI). pp. 552-558.

[4] Berman, P., Karpinski, M., Scott, A. D., 2003. Approximation hardness of short symmetric instances of MAX-3SAT. Electronic Colloquium on Computational Complexity (ECCC) (049).

[5] Brown, J., Minor, D. B., 2014. Selecting the best? spillover and shadows in elimination tournaments. Management Science, 3087-3102.

[6] Chatterjee, K., Ibsen-Jensen, R., Tkadlec, J., 2016. Robust draws in balanced knockout tournaments. In: Proceedings of the 24th International Joint Conference on Artificial Intelligence (IJCAI). AAAI Press, pp. 172179.

[7] Connolly, R. A., Rendleman, R. J., 2011. Tournament qualification, seeding and selection efficiency. Tech. Rep. 2011-96, Tuck School of Business.

[8] Edwards, C. T., 1996. Double-elimination tournaments: Counting and calculating. The American Statistician 50 (1), 27-33.

[9] Fomin, F. V., Kratsch, D., 2010. Exact Exponential Algorithms. Springer.

[10] Groh, C., Moldovanu, B., Sela, A., Sunde, U., 2012. Optimal seedings in elimination tournaments. Economic Theory 49 (1), 59-80. 
[11] Gusfield, D., Martel, C., 2002. The structure and complexity of sports elimination numbers. Algorithmica 32 (1), 73-86.

[12] Hazon, N., Aumann, Y., Kraus, S., Wooldridge, M., 2008. Evaluation of election outcomes under uncertainty. In: Proceedings of the 7th International Conference on Autonomous Agents and Multiagent Systems (AAMAS). pp. 959-966.

[13] Horen, J., Riezman, R., 1985. Comparing draws for single elimination tournaments. Operations Research 33 (2), 249-262.

[14] Huang, L., 2016. Prize and incentives in double-elimination tournaments. Economic Letters 147, 116-120.

[15] Kern, W., Paulusma, D., 2004. The computational complexity of the elimination problem in generalized sports competitions. Discrete Optimization 1 (2), 205-214.

[16] Kim, M. P., Suksompong, W., Vassilevska Williams, V., 2016. Who can win a single-elimination tournament? In: Proceedings of the 30th AAAI Conference on Artificial Intelligence (AAAI). pp. 516-522.

[17] Kim, M. P., Vassilevska Williams, V., 2015. Fixing tournaments for kings, chokers, and more. In: Proceedings of the 24th International Joint Conference on Artificial Intelligence (IJCAI). pp. 561-567.

[18] Lang, J., Pini, M. S., Rossi, F., Salvagnin, D., Venable, K. B., Walsh, T., 2012. Winner determination in voting trees with incomplete preferences and weighted votes. Journal of Autonomous Agents and Multi-Agent Systems 25 (1), 130-157.

[19] Lang, J., Pini, M. S., Rossi, F., Venable, K. B., Walsh, T., 2007. Winner determination in sequential majority voting. In: Proceedings of the 20th International Joint Conference on Artificial Intelligence (IJCAI). pp. 13721377 .

[20] Laslier, J.-F., 1997. Tournament Solutions and Majority Voting. SpringerVerlag.

[21] Marchand, E., 2002. On the comparison between standard and random knockout tournaments. Journal of the Royal Statistical Society 51 (2), 169178.

[22] Mattei, N., Walsh, T., 2016. Empirical evaluation of real world tournaments. CoRR abs/1608.01039.

URL http://arxiv.org/abs/1608.01039

[23] Rosen, S., 1986. Prizes and incentives in elimination tournaments. The American Economic Review 76 (4), 701-715. 
[24] Russell, T., van Beek, P., 2011. An empirical study of seeding manipulations and their prevention. In: Proceedings of the 22nd International Joint Conference on Artificial Intelligence (IJCAI). pp. 350-356.

[25] Russell, T., Walsh, T., 2009. Manipulating tournaments in cup and round robin competitions. In: Proceedings of the 1st International Conference on Algorithmic Decision Theory. pp. 26-37.

[26] Silver, N., March 2011. When 15th is better than 8th: The math shows the bracket is backward. On Five-ThirtyEight Blog, http://fivethirtyeight.blogs.nytimes.com/2011/03/15/when-15th-is-betterthan-8th-the-math-shows-the-bracket-is-backward/, last Accessed Feb. 2014.

[27] Stanton, I., Vassilevska Williams, V., 2011. Manipulating single-elimination tournaments in the Braverman-Mossel model. In: IJCAI Workshop on Social Choice and Artificial Intelligence.

[28] Stanton, I., Vassilevska Williams, V., 2011. Manipulating stochastically generated single-elimination tournaments for nearly all players. In: Proceedings of 7 th International Workshop on Internet and Network Economics (WINE). pp. 326-337.

[29] Stanton, I., Vassilevska Williams, V., 2011. Rigging tournament brackets for weaker players. In: Proceedings of the 22nd International Joint Conference on Artificial Intelligence (IJCAI). pp. 357-364.

[30] Stanton, I., Vassilevska Williams, V., 2013. The structure, efficacy, and manipulation of double-elimination tournaments. Journal of Quantitative Analysis in Sports 9 (4), 319-335.

[31] Tullock, G., 1980. Toward a Theory of the Rent-seeking Society. Texas A\&M University Press.

[32] Vassilevska Williams, V., 2010. Fixing a tournament. In: Proceedings of the 24th AAAI Conference on Artificial Intelligence (AAAI). AAAI Press, pp. 895-900.

[33] Vassilevska Williams, V., 2013. Fixing tournaments. In: Lipton, R. J., Regan, K. W., (Eds.), People, Problems, and Proofs : Essays From Gödel's Lost Letter: 2010. Springer, Ch. 61, pp. 319-322.

[34] Vu, T., Altman, A., Shoham, Y., 2009. On the complexity of schedule control problems for knockout tournaments. In: Proceedings of the 8th International Conference on Autonomous Agents and Multiagent Systems (AAMAS). pp. 225-232.

[35] Vu, T., Hazon, N., Altman, A., Kraus, S., Shoham, Y., Wooldridge, M., 2013. On the complexity of schedule control problems for knock-out tournaments. Working paper. 
[36] Vu, T., Shoham, Y., 2011. Fair seeding in knockout tournaments. ACM Transactions on Intelligent Systems Technology 3 (1), 1-17.

[37] Welsh, D. J. A., Merino, C., 2000. The Potts model and the Tutte polynomial. Journal of Mathematical Physics 41 (3), 1127-1152.

[38] Xia, L., Conitzer, V., 2011. Determining possible and necessary winners under common voting rules given partial orders. Journal of Artificial Intelligence Research 41 (2), 25-67. 